\title{
Magnetresonanztomografie bei Kindern in Sedierung - anästhesiologisches Prozedere, Zeitbedarf und Determinanten verzögerter Prozessabläufe
}

\author{
Anesthesiological Time Requirements and Reasons for Delayed Processes in MRI \\ Examinations of Children Undergoing Sedation or General Anesthesia
}

Autoren

Institute
S. Heinrich ${ }^{1}$, A. Irouschek¹, A. Ackermann ${ }^{1}$, T. Birkholz ${ }^{1}$, R. Janka², M. M. Lell ${ }^{2}$, J. Schmidt ${ }^{1}$

Anästhesiologische Klinik, Universitätsklinikum Erlangen

2 Radiologisches Institut, Universitätsklinikum Erlangen
Key words

- MR imaging

delayed processes

cost effectiveness

- clinical pathways

- hospital organisation and administration

eingereicht 30.10 .2012

akzeptiert $\quad 21.12 .2012$

\section{Bibliografie}

Dol http://dx.doi.org/

10.1055/s-0032-1330742

Online-Publikation: 21.3.2013

Fortschr Röntgenstr 2013; 185:

467-473 @ Georg Thieme

Verlag KG Stuttgart · New York .

ISSN 1438-9029

Korrespondenzadresse Herr Dr. Sebastian Heinrich Anästhesiologische Klinik, Universitätsklinikum Erlangen Krankenhausstraße 12

91054 Erlangen

Tel.: ++ 49/9131/8542474

Fax: ++49/9131/8536147

sebastian.heinrich@kfa.imed.

uni-erlangen.de

\section{Zusammenfassung \\ $\nabla$}

Ziel: Magnetresonanztomografie-Untersuchungen bei Kindern in Sedierung oder Allgemeinanästhesie stellen aufgrund der Vielzahl der beteiligten Disziplinen und der daraus entstehenden Schnittstellen eine besondere organisatorische Herausforderung für die beteiligten pädiatrischen, radiologischen und anästhesiologischen Abteilungen dar. Mit der vorliegenden Arbeit sollen Erkenntnisse über die Patientenstruktur, das anästhesiologische Vorgehen und die damit verbundenen Prozesszeiten im Zusammenhang mit MRT-Untersuchungen bei Kindern in Sedierung bzw. Allgemeinanästhesie gewonnen werden. Ziel ist es dabei, theoretische Möglichkeiten der Prozessoptimierung zu identifizieren und kritisch zu evaluieren.

Material und Methoden: Es erfolgte die retrospektive Auswertung und statistische Aufarbeitung der Anästhesieprotokolle von Kindern aller Altersgruppen, die sich in einer Universitätsklinik einem MRT in Sedierung oder Allgemeinanästhesie über eine Zeitspanne von 5 Jahren unterziehen mussten.

Ergebnisse: Die mediane Zeit für Ein- und Ausleitung bei Patienten ohne Atemwegssicherung betrug $6 \mathrm{~min}$ und war bei Patienten, die eine Larynxmaske (19 min) oder einen Endotrachealtubus (20 min) benötigten, signifikant länger $(p<0,001)$. Die Raten der notwendigen Atemwegssicherung betrugen bei Neugeborenen $67 \%$, bei Säuglingen $25 \%$, bei Kleinkindern $8 \%$ und bei Schulkindern 7\%. Durch verbesserte Patientenlogistik und eindeutige präinterventionelle organisatorische Absprachen hätten im Untersuchungszeitraum zusätzliche 69 h Untersuchungszeit mobilisiert werden können.

Schlussfolgerung: Die anästhesiologische Versorgung von Kindern im Neugeborenen und Kleinkindalter führt im Vergleich zur Versorgung von älteren Kindern zu einer Verlängerung der anästhesiologischen Prozesszeiten in der diagnosti-

\section{Abstract \\ $\nabla$}

Purpose: MRI examinations in children under sedation or general anesthesia are a multidisciplinary challenge for pediatric, radiology and anesthesiology departments. This article presents findings about patient population, anesthesiology procedures and process times in pediatric MRI procedures under sedation or general anesthesia. The analysis is focused on the potential to optimize process times.

Materials and Methods: Retrospectively over a 5-year period, data from diagnostic radiology procedures in children requiring sedation or general anesthesia were retrieved from anesthesia records and analyzed statistically.

Results: The median anesthesia time in patients without an airway device was $6 \mathrm{~min}$. In patients with a laryngeal mask (19 min) and in patients receiving endotracheal intubation (20 $\mathrm{min}$ ), the anesthesia time was significantly longer $(p<0.001)$. The need for airway management in the age groups was $67 \%$ for neonates, $25 \%$ for infants, $8 \%$ for toddlers and $7 \%$ for school children. Improved clinical pathways could potentially save additional time up to 69 hours in our period of investigation. Conclusion: In relation to older children, toddlers, infants and neonates had the longest process times in diagnostic radiology procedures, caused by the need to secure the airway. Improved preMRI processes have an enormous potential to generate additional examination time. 
schen Radiologie. Dies ist durch die häufigere Anwendung von Methoden zur Atemwegssicherung bei jüngeren Patienten bedingt. Das größte Potential zur Prozessoptimierung ist im präinterventionellen Bereich der Untersuchungen zu sehen.

\section{Einleitung}

\section{$\nabla$}

Die Anzahl der Untersuchungen mittels Magnetresonanztomografie (MRT) bei Kindern steigt in den letzten Jahren rasch an. Insbesondere onkologische Fragestellungen, seltene Erkrankungen und fragliche ZNS-Pathologien stehen dabei im Vordergrund $[1,2]$. Um bei diagnostischen radiologischen Untersuchen eine gute Untersuchungsqualität zu erreichen, sind die Kooperation und die Mitarbeit der Patienten von entscheidender Bedeutung. Dies gilt wegen der im Vergleich zur Computertomografie erheblich längeren Scanzeiten und der sich daraus ergebenden möglichen Bewegungsartefakte besonders für die Magnetresonanztomografie. Eine Kooperation im Sinne einer längeren Bewegungslosigkeit ist jedoch bei jungen Kindern meist nicht gegeben. Die räumliche Isolation, Trennung von der elterlichen Bezugsperson, unbekannte Umgebung, Lärm und Enge führen in der Regel zu einer Ablehnung der Untersuchung durch die Kinder und somit zu deren Undurchführbarkeit. Darüber hinaus ist auch bei älteren Kindern mit syndromalen Erkrankungen, die mit einer geistigen Retardierung einhergehen, keine Kooperation bei der Durchführung einer MRT zu erwarten. Die Gewinnung aussagekräftiger radiologischer Bilder ist in diesen Fällen nur mittels einer medikamentösen Sedierung möglich. Dies gilt insbesondere dann, wenn untersuchungsbedingt kurzzeitige Atempausen erforderlich sind, die sich nur mittels tiefer Sedierung/Narkose und Anwendung einer Atemwegssicherung erzielen lassen. Narkosearbeitsplätze in der Radiologie sind anästhesiologisch oft unstetig besetzt, befinden sich oft abseits vom anästhesiologischen Arbeitsumfeld im OP und sind gekennzeichnet durch einen ungewohnt eingeschränkten Zugang zum Patienten. Trotz der auf europäischer Ebene genormten Mindestausstattung eines Narkosearbeitsplatzes (EN 740) sind Medikamentenvorhaltung, Ausrüstung und personelle Unterstützung im Vergleich zum gewohnten Arbeitsbereich im OP oft nur in reduziertem Maße verfügbar [3]. Dem häufig geäußerten Wunsch zur Beschleunigung anästhesiologischer Prozesse stehen teilweise die Erfordernisse zur Notwendigkeit der Atemwegssicherung im Sinne der Patientensicherheit mit unzweifelhaft höherer Priorität entgegen. In diesem Zusammenhang sind die Ergebnisse einer früheren Untersuchung bedeutsam, welche die häufigsten anästhesieassoziierten Komplikationen nicht im OP, sondern bei sogenannten unstetigen Arbeitsplätzen, wie z. B. in der Radiologie oder in der Zahnheilkunde identifizierte [4]. Daher wird für unstete Sonderarbeitsplätze häufig die Einführung von „Sedation Teams“ gefordert, mit deren Hilfe die Raten an unerwünschten Ereignissen während diagnostischen Eingriffen gesenkt werden kann [5].

Ökonomische Aspekte sind beim Einsatz von medizinischen Großgeräten, wie MRT- Scannern, von großer Bedeutung. MRTScanner gehören zu den teuersten Medizinprodukten, über die ein Krankenhaus verfügt. Dabei spielen nicht nur die Investitionskosten von ca. 800000 - 1,5 Mio. $€$, sondern auch die laufenden Kosten, durch Wartungsverträge, Flüssiggasverbrauch, hohen Stromverbrauch und Personalkosten eine erhebliche Rolle [6]. Darüber hinaus fallen nicht unerhebliche Kosten für die Vorhaltung von MRT geeigneter Anästhesieausrüstung (Respirator,
Monitoringsysteme, Spritzenpumpen etc.) an. Diese Kosten können nur über eine entsprechende Auslastung amortisiert werden. Der daraus abgeleitete Wunsch nach möglichst kurzen anästhesiologischen Prozesszeiten ist daher verständlich.

In diesem Spannungsfeld zwischen möglicher Prozessoptimierung und unabdingbarer Patientensicherheit war es das Ziel der vorliegenden Untersuchung, im Sinne einer qualitätsgesicherten Medizin folgende Fragen zu beantworten:

1. Wie ist die Altersstruktur der Patienten, und welche anästhesiologischen Erfordernisse ergeben sich daraus?

2. Welchen Einfluss haben diese Unterschiede auf die Prozesszeiten und ist ein Optimierungspotential zu erkennen?

3. Wodurch entstehen Verzögerungen im Ablauf, und wären diese vermeidbar?

\section{Patienten und Methoden}

$\nabla$

In einem retrospektiven Qualitätsaudit wurden die Anästhesieakten aller Patienten gesichtet, die zwischen 15.5.2007 und 15.5.2012 im radiologischen Institut des Klinikums der Friedrich-Alexander Universität Erlangen-Nürnberg eine MRT-Untersuchung mit Anästhesiebeteiligung erhielten und die zum Zeitpunkt der Untersuchung jünger als 18 Jahre alt waren. Die Notwendigkeit eines Ethikvotums wurde von der örtlichen Ethikkommission mit Verweis auf den retrospektiven Charakter, die vollständige Anonymisierung sowie der abgeschlossenen Behandlung aller untersuchten Fälle verneint.

Nach Indikationsstellung zur diagnostischen MRT durch die anfordernde pädiatrische Abteilung wurden die Patienten von behandelnden Pädiatern vorbeurteilt und in der zentralen Anästhesieambulanz vorgestellt. Nach der präoperativen Visite in der zentralen Anästhesieambulanz erfolgte die Terminierung der MRT-Untersuchung durch die anfordernde pädiatrische Abteilung in Absprache mit dem radiologischen Institut. Während unaufschiebbar dringliche MRT-Untersuchungen jederzeit, auch im Bereitschaftsdienst, durchgeführt werden können, gibt es feste Terminkontingente für elektive MRT-Untersuchungen mit Anästhesiebeteiligung. $\mathrm{Zu}$ diesen Terminkontingenten wurde der anästhesiologische Sonderarbeitsplatz im MRT-Raum tageweise mit einem Fach- oder Oberarzt aus einem festen Personalstamm („Sedation Team“) der anästhesiologischen Klinik und einer dem Sedation Team zugehörigen Fachpflegekraft besetzt. Bei Fällen mit fraglicher Sedierungsindikation wurde die definitive Entscheidung zur Gabe eines Sedativums vom durchführenden Anästhesisten in Rücksprache mit den radiologischen Kollegen vor Beginn der MRT getroffen.

Die anästhesiologische Versorgung der pädiatrischen Patienten orientierte sich dabei an den Empfehlungen der Deutschen Gesellschaft für Anästhesie und Intensivmedizin. Abweichend von der genannten Empfehlung, wonach minimale und moderate Sedierungen bei Kindern mit ASA Status 1 oder 2 auch vom untersuchenden Radiologen durchgeführt werden könnten, wurden in unserer Untersuchung alle Sedierungen/Narkosen von Anästhesiefachärzten durchgeführt. Bei pädiatrischen Patienten, bei denen aufgrund der radiologischen Fragestellung einzelne MR- 


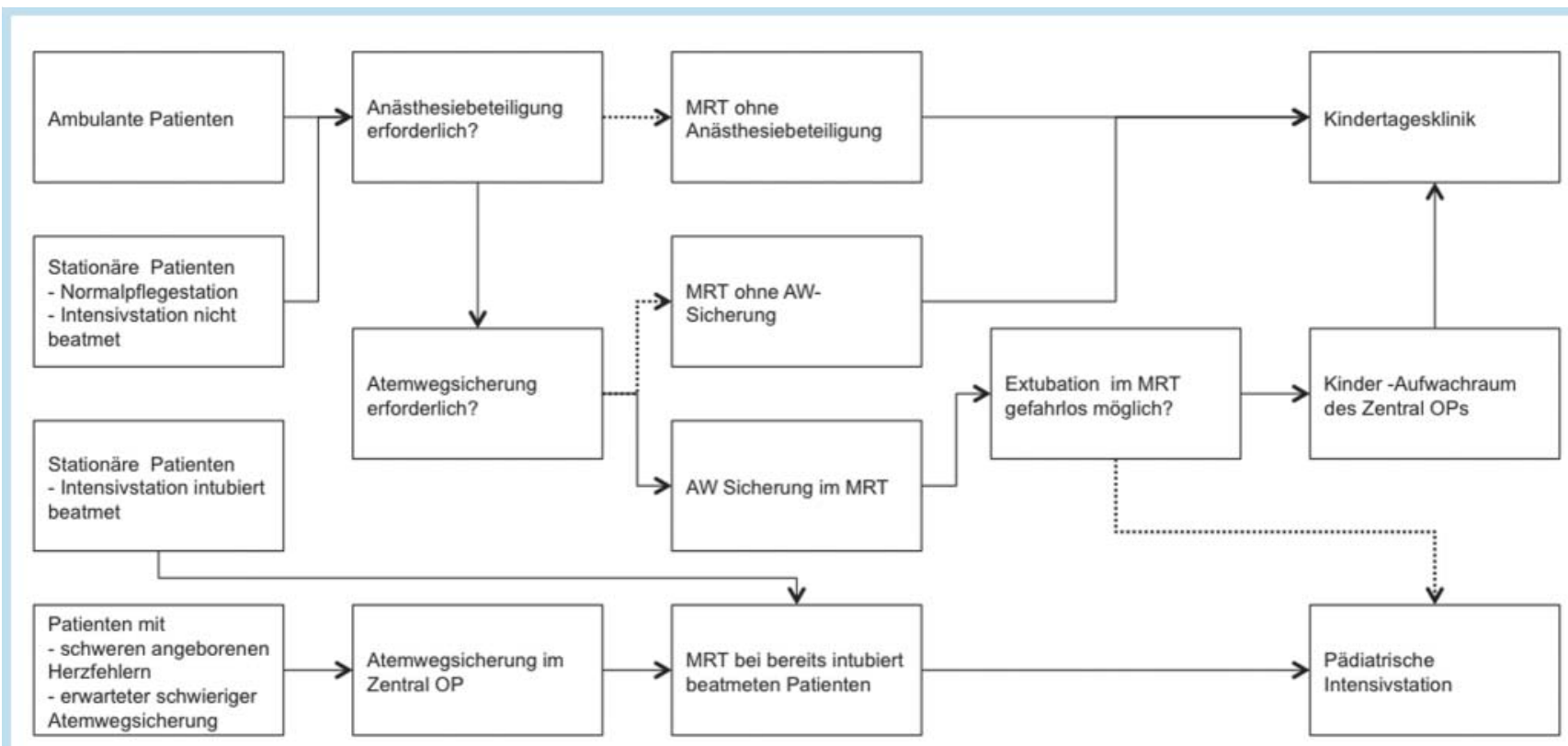

Abb. 1 Organigramm der Patientenströme.

Sequenzen in Apnoe erforderlich waren, wurde die Sedierung zu einer Narkose vertieft, sodass der Atemweg mit einer Larynxmaske oder einem Endotrachealtubus gesichert werden konnte. Diese Patienten wurden über die Atemwegssicherung druckkontrolliert beatmet. Um die Abläufe im MRT zu optimieren, wurde bereits vor dem in dieser Untersuchung erfassten Zeitraum in Absprache mit der anfordernden pädiatrischen Abteilung allen Kindern nach Aufnahme in der Tagesklinik der Kinderklinik ein i.v. Zugang gelegt. In Begleitung der Eltern und einer Pflegekraft der Kinderklinik wurden die Patienten dann ins MRT gebracht. Nach intravenöser Gabe eines Sedativums im Vorbereitungsraum erfolgte der Transport der Kinder auf die MRT-Liege im Scanraum. Die Sedierung wurde im MRT-Raum mittels kontinuierlicher Applikation von Propofol über eine Spritzenpumpe (Space Station MRI B.Braun ${ }^{\circledR}$, Melsungen) aufrechterhalten. Die Überwachung erfolgte mittels EKG, Sauerstoffsättigung und ggf. nicht invasiver Blutdruckmessung. Das endtidale $\mathrm{CO}_{2}$ wurde über eine Nasenbrille gemessen, bei der eines der Lumen zur Sauerstoffinsufflation und das zweite der beiden Lumen zur Messung des endtidalen $\mathrm{CO}_{2}$ diente. Dadurch können Apnoe-Episoden früher detektiert werden als mit der alleinigen Messung der Pulsoxymetrie [7, 8]. Als MRT-geeignetes Monitoringsystem diente der Precess Monitor (Invivo ${ }^{\circledR}$, Gainesville, USA). Die Beatmung wurde bei Patienten mit Larynxmaske oder Endotrachealtubus mit dem MRT geeigneten Narkoserespirator Fabius MRI (Dräger ${ }^{\circledR}$, Lübeck) bzw. vor dessen Verfügbarkeit mit einem Servo 900C (Siemens, Erlangen) sichergestellt.

Patienten der pädiatrischen Intensivstation, die bereits intubiert waren, wurden mit intensivärztlicher Begleitung ins MRT gebracht und dort vom Anästhesisten beatmet übernommen. Aspirationsgefährdete Kinder oder Kinder bei denen Apnoe-Sequenzen geplant waren, wurden im Vorraum des MRT vom Anästhesisten eingeleitet. $\diamond$ Abb. 1 zeigt das zugrunde liegende Flussdiagramm. Kinder mit komplexen Herzfehlbildungen, deren Anästhesieeinleitung und die damit verbundenen hämodynamischen Veränderungen ein hohes Risiko darstellen, oder Kinder mit Syndromen mit Gesichtsfehlbildungen und zu erwartender schwieriger Intu- bation wurden zum Teil im Anästhesiebereich des Kinderchirurgischen Zentrums des Zentral-OPs intubiert und von dort zum MRT transportiert. Nach Beendigung der Messung wurden beatmete Kinder von der pädiatrischen Intensivstation abgeholt. Kinder, die unmittelbar vor der Messung intubiert wurden und nach der Untersuchung im MRT extubiert wurden, konnten zur postoperativen Überwachung auf die pädiatrische Intensivstation oder den Kinderaufwachraum des Zentral-OPs verlegt werden. Zur retrospektiven anonymisierten Auswertung wurden alle elektronischen Narkoseprotokolle in eine Datenbank übertragen. Aus der Datenbank wurden die Art der Narkose und die applizierten Medikamente, die Art der Atemwegssicherung und die Prozesszeiten ausgewertet. Zur Auswertung erfolgte dabei die Gruppierung der Patienten in die verschiedenen u.g. Altersgruppen $1-5$ :

1. Neugeborene: Geburt bis 28 Tage

2. Säuglinge: 29 bis 364 Tage

- 3. Kleinkinder: 2. Lebensjahr bis 5. Lebensjahr

- 4. Schulkinder: 6. Lebensjahr bis 14. Lebensjahr

- 5. Jugendliche: 15. Lebensjahr bis Ende des 17. Lebensjahres

Die Einteilung des Gesundheitsstatus erfolgte nach dem 4-stufigen physical health status der American Society of Anesthesiologists (ASA). Dabei entspricht ASA 1 einem normalen gesunden Patienten, ASA 2 einem Patienten mit leichter Allgemeinerkrankung, ASA 3 einem Patienten mit schwerer Allgemeinerkrankung und ASA 4 einem Patienten mit einer oder mehreren schweren Allgemeinerkrankungen, die eine ständige Lebensbedrohung darstellen. Mit zunehmendem ASA-Status erhöht sich das Narkoserisiko. Zur Auswertung der Prozesszeiten wurden Datensätze mit unvollständiger Zeitdokumentation nicht berücksichtigt. Die statistische Auswertung erfolgte mit Statistica (StatSoft ${ }^{\circledR}$, Tulsa, USA). Neben elementarer Statistik wurden der Chi-Quadrat-Test und der t-Test mit einem festgelegten Signifikanzniveau von jeweils $\mathrm{p}=0,05$ verwendet.

Zur genaueren Analyse der tatsächlich aufgetretenen Verzögerungen im Ablauf wurde bei allen Datensätzen mit vollständiger Zeitendokumentation $(\mathrm{n}=1220)$ die Differenz aus der Präsenz- 


\begin{tabular}{|llcc} 
& $\begin{array}{l}\text { Gesamtverzögerung } \\
(\mathbf{m i n})\end{array}$ & $\begin{array}{l}\text { Median (25\%;75\%) } \\
(\mathbf{m i n})\end{array}$ & Anzahl \\
\hline verzögertes Bringen des Patienten vor der MRT & 2070 & $12(6 ; 29,3)$ & 100 \\
\hline Abholprobleme nach der MRT & 782 & $6,5(3 ; 13)$ & 70 \\
\hline unklares Prozedere in der MRT & 800 & $19(11 ; 25)$ & 29 \\
\hline verlängerte Anästhesiezeit z. B. durch AW-Sicherung & 707 & $9(3 ; 17,5)$ & 59 \\
\hline Wechsel von Sedierung zu AW-Sicherung & 382 & $26(9 ; 40)$ & 13 \\
\hline komplexe Einleitungen im OP (angeborene Herzfehler) & 1037 & $31(22 ; 49)$ & 25 \\
\hline paravenöser i. v. Zugang & 505 & $19(4,5 ; 34,8)$ & 18 \\
\hline gesamt & 6283 & $12(5 ; 26)$ & 314
\end{tabular}

Tab. 1 Analyse der Gründe für aufgetretene Verzögerungen bei Fällen mit vollständiger Zeitendokumentation $(n=1220)$ : Summe (min) pro gefundener Ursache, mediane Verzögerung ( $\mathrm{min}$ ) sowie unteres und oberes Quartil pro Fall einer Ursache, Anzahl der Fälle pro gefundener Ursache.

\begin{tabular}{|lcllc|} 
& Anzahl/Anteil (n/\%) & $\begin{array}{l}\text { mit AW-Sicherung } \\
\text { gesamt (n/\%) }\end{array}$ & $\begin{array}{l}\text { intubiert } \\
\text { gekommen (n/\%) }\end{array}$ & $\begin{array}{l}\text { AW-Sicherung im } \\
\text { MRT (n/\%) }\end{array}$ \\
\hline Neugeborene & $55 / 2335(2 \%)$ & $37 / 55(67 \%)$ & $34 / 55(62 \%)$ & $3 / 55(5,5 \%)$ \\
\hline Säuglinge & $327 / 2335(14 \%)$ & $82 / 327(25 \%)$ & $37 / 327(11 \%)$ & $45 / 327(14 \%)$ \\
\hline Kleinkinder & $1146 / 2335(49 \%)$ & $96 / 1146(8 \%)$ & $13 / 1146(1 \%)$ & $83 / 1146(7 \%)$ \\
\hline Schulkinder & $760 / 2335(33 \%)$ & $50 / 760(7 \%)$ & $3 / 760(0,4 \%)$ & $47 / 760(6 \%)$ \\
\hline Jugendliche & $47 / 2335(2 \%)$ & $7 / 47(15 \%)$ & $2 / 47(4 \%)$ & $5 / 42(11 \%)$ \\
\hline gesamt & $2335 / 2335(100 \%)$ & $272 / 2335(12 \%)$ & $89 / 2335(4 \%)$ & $183 / 2335(8 \%)$ \\
\hline
\end{tabular}

Tab. 2 Anzahl und Altersverteilung der Patienten mit Anästhesiebeteiligung sowie der Anteil an Patienten mit Atemwegssicherung (AW-Sicherung) insgesamt, bereits intubiert gekommene $\mathrm{Pa}$ tienten und Anteil der Patienten, bei denen die AW-Sicherung unmittelbar im MRT erfolgte.

zeit des Anästhesisten und der reinen radiologischen Scanzeit gebildet. Die mediane Differenz betrug dabei 26,5 min. Fälle, bei denen diese Differenz oberhalb der 3. Quartile (>31 min) lag, wurden hinsichtlich der gefundenen Verzögerungen im Ablauf gesondert und ausführlich überprüft. Bei der genaueren Überprüfung dieser Protokolle wurde die Verzögerung einer der in der Tab. 1 genannten Gründe zugeordnet. Wurde in einem Protokoll mehr als ein Grund für die aufgetretene Verzögerung gefunden, wurde dem Datensatz nur der Grund für die längste Verzögerung zugeordnet. Die Verzögerungen errechneten sich dabei aus der Differenz der Präsenzzeit des Anästhesisten und der Scanzeit. Jede Minute über $31 \mathrm{~min}$ dieser Differenzzeit wurde dabei als Verzögerung gewertet.

\section{Ergebnisse \\ $\nabla$}

Insgesamt wurden 2335 MRT-Untersuchungen mit Anästhesiebeteiligung bei 1710 Patienten unter 18 Jahren im Untersuchungszeitraum durchgeführt. Die dabei dokumentierten Hauptdiagnosen wurden den in 0 Abb. 2 gezeigten Kategorien zugeordnet. In 66 Fällen (3\%) wurde eine dringliche Indikation oder eine Notfallindikation zum MRT gestellt, 2270 Fälle waren (97\%) elektiv geplante Untersuchungen. Das Geschlechterverhältnis betrug 60/40\% (m/w), das mediane Alter 3,5 Jahre. Die Verteilung der einzelnen Altersklassen sowie der Anteil an Interventionen, bei denen eine Atemwegssicherung notwendig war, zeigt Tab. 2. In 272 Fällen (= $12 \%$ aller Untersuchungen) erfolgte eine kontrollierte Beatmung über eine Larynxmaske oder einen Endotrachealtubus. Bei 183 (67\%) der beatmeten Patienten erfolgte die Atemwegssicherung durch Anästhesisten, davon in 24 dieser Fälle (13\%) im Rahmen komplexer Narkoseeinleitungen im OP. 89 Patienten (33\%) wurden von der pädiatrischen Intensivstation bereits intubiert und beatmet zum MRT gebracht. Bei Kindern unter 1 Jahr (Neonaten und Säuglinge) musste signifikant häufiger eine Atemwegssicherung durchgeführt werden als bei Kindern, die älter als 1 Jahr waren (Kleinkinder, Schulkinder und Jugendliche) ( $p<0,001)$. 44\% der Kinder (119 von 272) mit notwendiger Atemwegssicherung waren jünger als 1 Jahr (Neo-

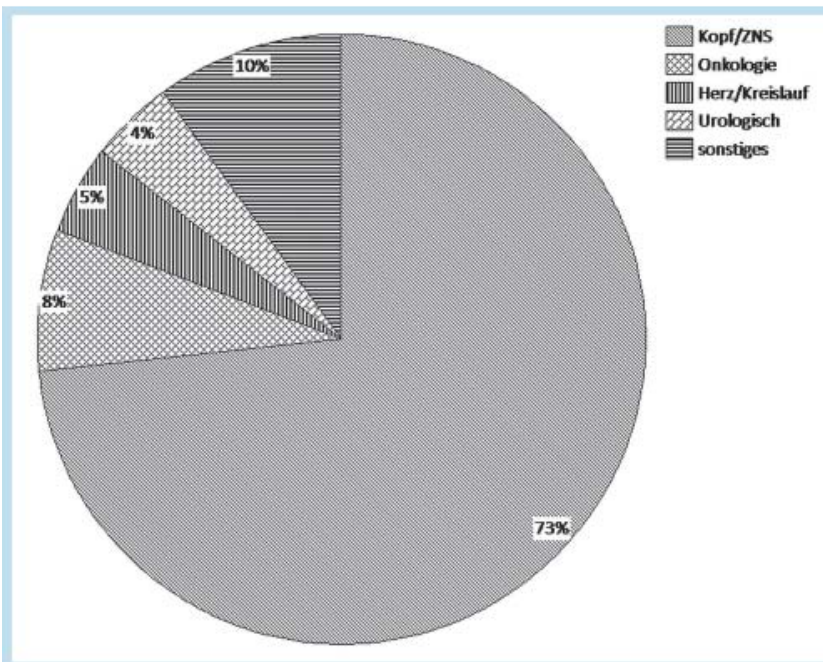

Abb. 2 Zuordnung der dokumentierten Hauptdiagnosen.

naten, Säuglinge). Diese beiden Altersgruppen wiesen signifikant häufiger einen ASA-3- oder ASA-4-Status auf als Kinder der zusammengefassten Altersgruppen 3 - 5 (32 vs. 19\%; p < 0,001). Bei 19 Patienten (1\%) erwies sich die Sedierungstiefe als unzureichend, sodass in der Folge die Sedierung vertieft und der Atemweg gesichert werden musste. Bei 9 dieser Patienten musste eine Atemwegssicherung durchgeführt werden, weil es unter Spontanatmung zu Bewegungen des Kopfes mit konsekutiver Einschränkung der Untersuchungsqualität durch Bewegungsartefakte kam. Bei 7 Patienten trat infolge einer notwendigen Vertiefung der Sedierung eine insuffiziente Spontanatmung auf, die mit der Notwendigkeit einer Atemwegssicherung und einer kontrollierten Beatmung einherging. Bei 3 Patienten musste im Rahmen von Zwischenfällen eine notfallmäßige Atemwegssicherung erfolgen.

Ein Patient erlitt einen generalisierten Krampfanfall während der Messung, ein zweiter Patient eine anaphylaktische Reaktion nach Gabe des Kontrastmittels, bei einem dritten Patienten kam es 
Tab. 3 Prozesszeiten (min) dargestellt als Median (unteres Quartil; oberes Quartil) bei allen Patienten, Patienten ohne AW-Sicherung, bei Patienten, die intubiert übernommen wurden und Patienten, die im MRT mit einer Larynxmaske oder einem Endotrachealtubus versorgt wurden.

\begin{tabular}{|lccccc} 
& alle Patienten & $\begin{array}{l}\text { ohne AW- } \\
\text { Sicherung }\end{array}$ & $\begin{array}{l}\text { intubiert } \\
\text { übernommen }\end{array}$ & mit Larynxmaske & mit Tubus \\
\hline vollständige Zeitendokumentation & $1348 / 2335(58 \%)$ & $1159 / 2062(56 \%)$ & $49 / 89(55 \%)$ & $70 / 93(75 \%)$ & $70 / 91(77 \%)$ \\
\hline medianes Alter & 3,5 Jahre & 3,7 Jahre & 48 Tage & 3,8 Jahre & 1,5 Jahre \\
\hline Einleitungszeit $(\min )$ & $4(3 ; 7)$ & $4(3 ; 6)$ & $9(5 ; 13)$ & $10(8 ; 15)$ & $14(10 ; 21)$ \\
\hline Radiologiezeit $(\min )$ & $49(39 ; 60)$ & $47(38 ; 58)$ & $50(44 ; 62)$ & $68(54 ; 86)$ & $60(48 ; 83)$ \\
\hline Scanzeit (min) & $45(36 ; 56)$ & $44(34 ; 54)$ & $48(40 ; 57)$ & $63(49 ; 80)$ & $56(42 ; 75)$ \\
\hline Ausleitungszeit $(\min )$ & $3(1 ; 4)$ & $2(1 ; 4)$ & $2(1 ; 4)$ & $9(5 ; 14)$ & $6(3 ; 11)$ \\
\hline
\end{tabular}

nach einer Hustenepisode zu einem Laryngospasmus. Ein weiterer Patient benötigte eine Kompartmentspaltung nach paravenöser Infusion. Bei allen Patienten fanden sich retrospektiv nach Einsicht in die Krankenakten keine Hinweise für bleibende funktionelle Beeinträchtigungen. Sonstige schwere Komplikationen und Zwischenfälle traten nicht auf. Bei 2268 Fällen (97\%) wurde Propofol als Hypnotikum eingesetzt. 70-mal (3\%) wurde zusätzlich zum Hypnotikum noch ein Opiat verabreicht, wobei jedoch 5 Patienten sowohl Fentanyl als auch Remifentanil erhielten. Die dabei eingesetzten Substanzen waren Fentanyl $(n=38)$, Remifentanil $(n=23)$ und Alfentanil $(n=9) .55$ der Opiatgaben $(79 \%)$ erfolgten bei Patienten, die eine Allgemeinanästhesie mit Atemwegssicherung erhielten.

Zur Auswertung der Prozesszeiten konnten 1348 der 2335 (58\%) Datensätze mit vollständig dokumentierten Zeiten herangezogen werden. Die dabei erhobenen Prozesszeiten zeigt $\bullet$ Tab. 3. Die Einleitungszeit beschreibt dabei die Zeit von Beginn der anästhesiologischen Maßnahmen bis zu deren vorläufigem Ende bzw. dem Transfer in den MRT-Messraum. Mit dem Ende der Einleitungszeit beginnt die Radiologiezeit, sie endet nach dem Transfer aus dem Messraum in den Vorbereitungsraum und beinhaltet darüber hinaus auch die reine Messzeit. Mit dem Ende der Radiologiezeit beginnt die Ausleitungszeit und endet zu dem Zeitpunkt, an dem der Patient abgeholt werden kann.

Patienten, die im Anschluss an die MRT extubiert worden sind, wiesen eine mediane Ausleitungszeit von 9 min auf. Patienten, die intubiert verlegt worden sind, hatten eine mediane Ausleitungszeit von $2 \mathrm{~min}(\mathrm{p}<0,001)$. Die genauere Analyse der tatsächlich aufgetretenen Verzögerungen im Ablauf bei allen Datensätzen mit vollständiger Zeitendokumentation $(n=1348)$ sind in - Tab. 1 dargestellt. Verzögerungen beim Patiententransport summierten sich auf $2854 \mathrm{~min}$ ( $48 \mathrm{~h}$ ), davon entfielen $2070 \mathrm{~min}$ $(35 \mathrm{~h})$ auf nicht rechtzeitiges Bringen der Patienten zum MRT und $782 \mathrm{~min}(13 \mathrm{~h})$ auf verzögertes Abholen der Patienten nach Beendigung der MRT. Organisatorische Mängel, deren Klärung unmittelbar präinterventionell umfangreiche Rücksprachen und Telefonate notwendig machten, führten zu einer kumulativen Verzögerung von $800 \mathrm{~min}$ (13 h). Durch Venenzugänge, die nach dem Transfer zur MRT-Einheit bereits paravenös waren, entstand eine kumulative Verzögerung von $505 \mathrm{~min}(8 \mathrm{~h})$. Alle genannten Verzögerungen summierten sich in unserer Untersuchung auf $4159 \min (69 \mathrm{~h})$.

\section{Diskussion}

$\nabla$

In unserem Kollektiv von pädiatrischen Patienten, die sich einer MRT-Untersuchung in Sedierung/Narkose unterziehen mussten, stellten die Gruppe der Kleinkinder mit 49\% und die Gruppe der
Schulkinder mit 33\% den Hauptanteil der Patienten dar. Auf die Gruppe der Jugendlichen entfielen hingegen nur $2 \%$ der Fälle. In unserem Kollektiv wiesen Neonaten und Säuglinge (Altersgruppen 1 und 2) die höchste Rate in der Notwendigkeit einer Atemwegssicherung auf. Obwohl diese Altersgruppen in der Summe nur $16 \%$ aller Patienten mit Anästhesiebeteiligung darstellten, entfielen auf sie $44 \%$ der Atemwegssicherungen. Da jedoch gerade bei Kindern unter $1 \mathrm{Jahr}$, die Atemwegssicherung häufig schwieriger sein kann, als bei älteren Kindern [9], ist es notwendig, dass alternative Atemweghilfen und entsprechendes Gerät zum Beherrschen des schwierigen Atemwegs auch für kleine Kinder entweder stationär im MRT vorgehalten wird oder bei tageweiser Besetzung des Anästhesiearbeitsplatzes im MRT von dem Anästhesieteam mitgebracht wird. Die dabei zu beachtenden generellen Anforderungen für strukturelle, personelle und organisatorische Abläufe bei der Durchführung von Sedierungen bei pädiatrischen Patienten werden in einer Handlungsempfehlung von der Deutschen Gesellschaft für Anästhesie und Intensivmedizin dargelegt [10]. In dieser Handlungsempfehlung wird auch auf die unterschiedlichen Anforderungen in Abhängigkeit vom Alter der Kinder hingewiesen.

Die erhobenen Daten zeigen, dass der notwendige anästhesiologische Zeitbedarf an den Gesamtprozesszeiten bei MRT-Untersuchungen von Kindern in Sedierung stark von der Erfordernis einer durchgeführten Atemwegssicherung abhängig ist. Die Indikationen zur Sicherung des Atemwegs lassen sich dabei in 2 Gruppen gliedern. Anästhesiologische Indikationen stellen die Aspirationsgefahr bei fehlender Nüchternheit oder einer Grunderkrankung mit Störung der Magendarmpassage und die Assistierung einer unzureichenden Spontanatmung dar. Die Hauptindikation aus radiologischer Sicht zur Atemwegssicherung stellte eine MRT-Untersuchung innerer Organe mit der Notwendigkeit von Atemmanövern/Atemstopps dar. Aufgrund der höheren Komplexität dieser Untersuchungen sind bei beatmeten Patienten nicht nur die anästhesiologischen, sondern auch die radiologischen Prozesszeiten verlängert. Den klinischen Erfordernissen respektive der Sicherung von potenziell gefährdeten Vitalfunktionen ist dabei unbestritten Vorrang vor optimierten Prozesszeiten und ökonomischen Erwägungen einzuräumen. Andererseits lassen sich auch die geforderten Apnoesequenzen ohne Atemwegssicherung nicht realisieren. Die Atemwegssicherung ist daher keine vermeidbare Verzögerung, sondern unabdingbare klinische Notwendigkeit und daher kein geeignetes Feld, um die Gesamtprozesszeiten zu verkürzen. Unabhängig davon fällt anästhesiebedingt lediglich $1 / 3$ des gesamten Zeitbedarfs an. Letztendlich ist jedoch davon auszugehen, dass Untersuchungen von Kindern, unabhängig ob sie nun mit oder ohne Anästhesiebeteiligung sind, ein größeres Maß an organisatorischer Unsicherheit bieten, als dies bei Erwachsenen der Fall ist. Das Ziel sollte dabei 
dennoch eine effiziente Prozessgestaltung sein, um eine optimale Auslastung von MRT und Anästhesie zu gewährleisten.

Als zur Prozessoptimierung am geeignetsten erscheinen die Patientenlogistik und die Verbesserung organisatorischer Absprachen. So sind 2/3 der in $\bullet$ Tab. 1 aufgeführten Gesamtverzögerung von $6283 \mathrm{~min}(105 \mathrm{~h})$ vermeidbar, ohne dass dabei sicherheitsrelevante Aspekte der Patientenversorgung berührt würden. Durch pünktliches Bringen und Abholen der Patienten, konsequentes Anlegen und Sichern eines i.v. Zugangs und der Abklärung offener Fragen zu der gewünschten Untersuchung oder der MRT Kompatibilität von Implantaten bereits im Vorfeld der geplanten Untersuchung entstanden im Zeitraum unserer Studie unnötige Leerlaufzeiten für MRT und Anästhesie von $69 \mathrm{~h}$, also ca. 2 Arbeitswochen. Legt man die oberen Quartilen-Werte der vermeidbaren Verzögerungen eines nicht brauchbaren i.v. Zugangs (35 min) und eines verzögerten Beginns durch verspäteten Transport zum MRT (29 min) zugrunde, so ergibt sich bereits bei Eintreten dieser zweier Ereignisse an einem Untersuchungstag eine kumulierte Verzögerung, die theoretisch eine weitere MR-Untersuchung in Sedierung ohne Atemwegssicherung (Radiologiezeit $44 \mathrm{~min}+$ Anästhesiezeit $6 \mathrm{~min}$ ) ermöglicht hätte. Um auch kurzfristig entstehende Leerzeiten zu reduzieren, sollten ambulante Patienten rechtzeitig in eine holding area einbestellt werden, um frei werdende Kapazitäten rasch nutzen zu können. Eine solche rasche Nutzung kurzfristig frei werdender MR-Kapazitäten ist wie die gesamte Logistik jedoch stark von den örtlichen Gegebenheiten und dem Untersuchungsaufkommen abhängig, und kann hier nur spezifisch für die hiesigen Gegebenheiten betrachtet werden. $\mathrm{Zu}$ beachten ist außerdem, dass Untersuchungen von einbestellten und nüchtern gelassenen pädiatrischen Patienten in aller Regel nicht ausfallen, sondern notfalls auch verspätet durchgeführt werden. Dadurch kommt es jedoch auch bei Untersuchungen, die nach dem pädiatrischen Untersuchungsblock geplant sind, zu Verzögerungen und ggf. auch Untersuchungsausfällen.

Obwohl die Magnetresonanztomografie ein risikoarmes Verfahren der bildgebenden Diagnostik darstellt, sollte jedoch ein unmittelbares anästhesiologisches Risiko durch die Sedierung/ Allgemeinanästhesie nicht außer Acht gelassen werden, das abhängig von den Vorerkrankungen des Kindes erheblich sein kann $[4,5]$. Darüber hinaus mehren sich Hinweise auf anästhetikaassoziierte neurotoxische Langzeiteffekte bei Säuglingen und Kleinkindern nach Allgemeinanästhesien [11-13]. So konnten mehrere aktuelle Studien aufzeigen, dass wiederholte Narkosen vor Erreichen des vierten Lebensjahres mit späteren Lernschwierigkeiten assoziiert sind. Daher sind bei jeder MRT-Untersuchung, insbesondere bei Wiederholungsuntersuchungen, die möglichen Risiken mit den therapeutischen Konsequenzen, die sich aus der diagnostischen Untersuchung ergeben, kritisch abzuwägen. Vor diesem Hintergrund sowie dem Ergebnis unserer Untersuchung, wonach Patienten unter einem Jahr signifikant häufiger eine Atemwegssicherung mit allen damit verbunden Risiken benötigten, wäre zu diskutieren, ob aufschiebbare Untersuchungen bei Kindern nicht erst zu einem späteren Zeitpunkt durchgeführt werden. So ließe sich das daraus hervorgehende Risiko sowohl hinsichtlich Atemwegskomplikationen bzw. Atemwegsmanagement und potenzieller Neurotoxizität der zur Sedierung notwendigen Anästhetika deutlich reduzieren. Auch technische Neuerungen können zur Reduktion der Anzahl von Sedierungen sowie den damit verbundenen Risiken beitragen. So konnte die Notwendigkeit einer Sedierung für Schädel-MRTs bei Kindern in einer Machbarkeitsstudie mit dem Einsatz von
BLADE-Sequenzen deutlich reduziert werden, ohne dass dabei nennenswerte Abstriche der Bildqualität in Kauf genommen werden mussten [14]. Auch die einzeitige Ganzkörper-MRT stellt im Gegensatz zu Mehrfachuntersuchungen an Körperteilen und Organsystemen eine entsprechende Risikoreduktion dar [15].

Die vorliegende Studie weist vor allem aufgrund des retrospektiven Charakters einige Limitationen auf. So beruhen alle Ergebnisse auf der Basis subjektiv durchgeführter Dokumentation in den Narkoseprotokollen. Die Dokumentation in den elektronischen Narkoseprotokollen ist in erster Linie für operative Eingriffe konzipiert und wurde nicht auf die speziellen Gegebenheiten einer MRT-Untersuchung angepasst. So erfolgte beispielsweise die Dokumentation der reinen Scanzeit als „Schnitt-Naht“-Zeit. Da in einigen Fällen eine solche Dokumentation nicht oder uneinheitlich erfolgte, jedoch nur vollständige Datensätze ausgewertet wurden, konnten zur Erhebung der Prozesszeiten nur 1220 (58\%) der 2099 Datensätze zur Auswertung herangezogen werden.

\section{Schlussfolgerungen \\ $\nabla$}

Die erhobenen Daten zeigen, dass die anästhesiologischen Prozesszeiten bei MRT-Untersuchungen bei Kindern im Wesentlichen von der Notwendigkeit einer Atemwegssicherung bestimmt werden. Die Notwendigkeit einer Atemwegssicherung ist bei Kindern unter einem Jahr signifikant häufiger notwendig gewesen als bei älteren Patienten. Dem muss im Sinne der Patientensicherheit durch die Bereitstellung von in der Kinderanästhesie erfahrenen Anästhesisten und entsprechender Ausstattung des Narkosearbeitsplatzes Rechnung getragen werden. Die präinterventionelle Vorbereitung zeigt ein großes Potenzial zur Prozessoptimierung, ohne dass dabei Abstriche bei der Patientensicherheit in Kauf genommen werden müssen. Eine konsequente Vorbereitung der Patienten bestehend aus der Aufklärung der erforderlichen Nüchternheitsgrenzen, Abklärung der MRT-Gängigkeit von Implantaten, eindeutig festgelegtem Prozedere, einem rechtzeitigen Einbestellen des Patienten, vorzeitiger Anlage und Sicherung eines Venenzugangs sowie pünktlichem Transfer zum MRT und zurück reduzieren vermeidbare Verzögerungen und führen darüber hinaus zu einer erhöhten Patientensicherheit.

\section{Literatur}

1 Reiss-Zimmermann M, Sorge I, Sterker I et al. MR imaging of orbital disorders in paediatric patients. Fortschr Röntgenstr 2011; 183: 781 787

2 Schwenzer NF, Pfannenberg C, Reischl G et al. Application of MR/PET in oncologic imaging. Fortschr Röntgenstr 2012; 184: 780 - 787

3 Deutsche Gesellschaft für Anästhesiologie und Intensivmedizin und Bund Deutscher Anästhesisten. Ausstattung des anästhesiologischen Arbeitsplatzes. Anästh Intensivmed 2013; 54: 39-42

4 Cote CJ, Notterman DA, Karl HW et al. Adverse sedation events in pediatrics: a critical incident analysis of contributing factors. Pediatrics 2000; 105: 805-814

5 Cravero JP, Beach ML, Blike GT et al. The incidence and nature of adverse events during pediatric sedation/anesthesia with propofol for procedures outside the operating room: a report from the Pediatric Sedation Research Consortium. Anesth Analg 2009; 108: 795-804

6 May B. Überlegungen zum wirtschaftlichen Betrieb einer Kinderradiologie. Kind und Radiologie 2009; 22: 38-48

7 Yldzdas D, Yapcoglu H, Ylmaz HL. The value of capnography during sedation or sedation/analgesia in pediatric minor procedures. Pediatr Emerg Care 2004; 20: 162 - 165

8 Anderson JL, Junkins E, Pribble C et al. Capnography and depth of sedation during propofol sedation in children. Ann Emerg Med 2007; 49: $9-13$ 
9 Heinrich S, Birkholz T, Ihmsen $\mathrm{H}$ et al. Incidence and predictors of difficult laryngoscopy in 11.219 pediatric anesthesia procedures. Paediatr Anaesth 2012; 22: 729-736

10 Philippi-Höhne CBK, Wulff B, Schmitz B et al. Analgosedierung für diagnostische und therapeutische Maßnahmen im Kindesalter. Anästhesie und Intensivmedizin 2010; 51: $603-614$

11 Stratmann G. Review article: Neurotoxicity of anesthetic drugs in the developing brain. Anesth Analg 2011; 113: 1170-1179
12 Vutskits L. Anesthetic-related neurotoxicity and the developing brain: shall we change practice? Paediatr Drugs 2012; 14: 13-21

13 Reddy SV. Effect of general anesthetics on the developing brain. J Anaesthesiol Clin Pharmacol 2012; 28: 6-10

14 Heyer CM, Lemburg SP, Sterl S et al. Dispensing with Sedation in Pediatric MR Imaging of the Brain: What is Feasible? Fortschr Röntgenstr 2012; 184: $1034-1042$

15 Schaefer JF, Kramer $U$. Whole-body MRI in children and juveniles. Fortschr Röntgenstr 2011; 183: 24-36 